\title{
Caracterización de alteraciones en la molécula de hemoglobina en afrodescendientes colombianos
}

\author{
Characterization of molecular alterations of hemoglobin in \\ Afro-Colombians
}

Martha Castillo ${ }^{1}$ Ana Lucia Oliveros ${ }^{1}$

\section{Resúmen}

Objetivo: Caracterizar alteraciones de la molécula de hemoglobina en afrodescendientes entre 18 a 50 años, a través de hemograma, estudio de sangre periférico, proteína $C$ Reactiva, ferritina sérica, receptor soluble de transferrina sRTf y electroforesis de Hemoglobina. Materiales y métodos: Esta investigación contó con un total de 56 muestras: 10 hombres y 46 mujeres. Resultados: La población en estudio perteneciente al Distrito de Aguablanca en la Ciudad de Cali presentó alteraciones en la cadena de hemoglobina asociada a Hemoglobina S, Hemoglobina $\mathrm{C}$ y posible talasemias por presencia de Hemoglobina Fetal y Hemoglobina A2 aumentadas. También se presentaron deficiencias de hierro, aunque en baja proporción, en diferentes estadios con respecto a la población general.

Palabras clave: afrodescendiente, hemoglobinopatías, ferropenias, anemias.

\begin{abstract}
Objective: Characterize molecular alterations of hemoglobin in Afro-Colombian subjects, ranging in age from 18 to 50 years. Analyses were conducted via complete blood count, peripheral blood smear, C-Reactive Protein, serum Ferritin, Soluble Transferrin Receptor (sTfR) and electrophoresis. Materials and Methods: A total of 56 subjects were analyzed, 10 men and 46 women. Results: Subjects (from the Aguablanca District in the City of Cali) showed alterations in the hemoglobin chain associated with Hemoglobin S, Hemoglobin $\mathrm{C}$ and possible thalassemias due to Fetal Hemoglobin and augmented Hemoglobin A2. Additionally, a small incidence of subclinical deficiencies of iron at different stages was also detected.
\end{abstract}

Keywords: Afro-Colombian, Hemoglobinopathies, iron deficiencies, anemias.

' Docentes- Investigadoras Universidad Colegio Mayor de Cundinamarca, Bogotá, D.C. Colombia. Corresponcencia:mlcastillo@unicolmayor.edu.co 


\section{Introducción}

En los últimos años en Colombia se ha incrementado la población desplazada en diferentes regiones, esto origina que el intercambio genético sea mayor y por consiguiente enfermedades como la anemia de células falciformes, combinada con la anemia ferropénica, se presenten en regiones que habitualmente no son de alta incidencia. (1-3).

En Colombia, las anemias que predominan son las ferropénicas, se calcula que cerca del $30 \%$ de población afrodescendientes la padecen. Considerando las alteraciones que se puede presentar por deficiencia de hierro es importante la detección temprana de éstas a fin de instaurar el tratamiento oportuno y dirigir campañas de prevención a la población por medio de educación a la comunidad (4-5).

Las alteraciones en la cadena de hemoglobina, genéticamente sigue el curso de las leyes de Mendel, manifestándose de manera homocigota con sintomatología característica y de manera heterocigota presentándose en individuos portadores con rasgo asociado a otras alteraciones ( $\mathrm{HbS}, \mathrm{Hb} \mathrm{C}, \mathrm{Hb} \mathrm{A}$, Talasemias) (6-8). Dichas alteraciones tienen una incidencia directa en población afrodescendiente generando un incremento en la morbi-mortalidad de este grupo poblacional.

\section{Materiales y métodos}

Las muestras utilizadas correspondieron a hombres y mujeres afrodescendientes, habitantes de la costa pacífica colombiana, quienes cumplían los criterios de inclusión. El proyecto fue inicialmente presentado al líder de la comunidad informando la importancia de la investigación y las condiciones para la recolección de la muestra, todos los participantes fueron voluntarios y firmaron el consentimiento informado. Un total de 56 muestras de sangre total y suero fueron recolectadas para realizar hemograma automatizado, estudio de sangre periférica y electroforesis de hemoglobina, proteína $\mathrm{C}$ reactiva.

El análisis descriptivo consistió en determinar las variables según sus porcentajes; en las variables continuas se analizó la distribución aplicando la prueba de Shapiro-Wilk (para muestras menores de 100 datos), en las variables con distribución normal se presenta el promedio y la distribución estándar, y en las variables con distribución no normal se describen según la mediana $(\mathrm{Md})$ y el rango inter-cuartíleo (RI).

Todas las variables fueron convertidas a proporción usando SPSS 19.0. Todas las variables numéricas fueron categorizadas de acuerdo a los criterios clínicos.

\section{Resultados}

El estudio involucró población afrodescendiente de la costa pacífica, habitantes Del Distrito de Agua Blanca de la ciudad de Cali, Colombia, 56 muestras fueron recolectadas de las cuales 10 (17.8\%) correspondían a hombres y 46 (82.2\%) a mujeres.

\section{Presencia de anemia}

Según los valores biológicos de referencia de la OMS se encontró que el 11.1\% (1/10) de los hombres y el 4.3\% (2/46) de las mujeres, presentan anemia. Se observa que las mujeres presentan valores de hemoglobina menores que los hombres, como se reporta en la literatura, Tabla 1. El porcentaje de anemia se presenta mayor en la población masculina. 
Tabla 1. Porcentaje de anemia en la muestra estudiada.

\begin{tabular}{lcccc} 
Localidad & Sex & Anemia & $\begin{array}{c}\text { Total } \\
\text { muestras }\end{array}$ & $\begin{array}{c}\text { Porcentaje } \\
\text { con anemia }\end{array}$ \\
\hline Cali & M & 1 & 10 & $11.1 \%$ \\
& F & 2 & 46 & $4.3 \%$ \\
Total & & $\mathbf{3}$ & $\mathbf{5 6}$ & $\mathbf{5 . 4 \%}$ \\
\hline
\end{tabular}

\section{Volumen corpuscular medio VCM}

En los hombres se encontró un $20 \%(2 / 10)$ y en las mujeres $4.3 \%$ (2/46) de VCM bajo, lo que indica la presencia de glóbulos rojos microcíticos, Tabla 2 .

Tabla 2. Volumen Corpuscular medio (VCM) en la muestra estudiada.

\begin{tabular}{lcc} 
VCM & Hombre & Mujer\% \\
\hline Normal & 80 & 95,7 \\
Bajo & 20 & 4,3 \\
\hline
\end{tabular}

\section{Proteina C Reativa (PCR)}

De la población total, el $14.3 \%(8 / 56)$ son positivos para PCR, mientras el $85.7 \%$ (48/56) son negativas, esto indica que 8 de las participantes en el estudio tienen probablemente procesos inflamatorios o infecciosos, Tabla 3.

Tabla 3. Resultados de la Proteína C Reactiva en la muestra estudiada.

\begin{tabular}{|c|c|}
\hline PCR & \\
\hline Negativo & $85,7 \%$ \\
\hline Positivo & $14,3 \%$ \\
\hline
\end{tabular}

\section{Ferritina}

La población masculina no presentó niveles de ferritina bajos, mientras que el 32\% (15/46) mujeres presentaron valores de ferritina bajas, lo que se relaciona con depósitos de hierro disminuidos, Tabla 4.
Tabla 4. Resultados de la prueba de Ferritina en la muestra estudiada.

\begin{tabular}{lcc} 
Ferritina & Hombre $\%$ & Mujer \% \\
\hline Normal & 100 & 68 \\
Baja & 0 & 32 \\
\hline
\end{tabular}

\section{Receptor Soluble de Transferrina sTfR}

El 10\% (1/9), de la población masculina presentó sTfR elevado y el $26 \%$ (12/44) de la población femenina presentó sTfR, Tabla 5, el aumento del receptor soluble de transferrina se relaciona con necesidades de hierro para la síntesis de hemoglobina.

Tabla 5. Resultados de la prueba de Receptor Soluble de Transferrina sTfR en la muestra estudiada.

\begin{tabular}{lcc}
\hline sTfR & Hombre $\%$ & Mujer \% \\
\hline Normal & 90 & 74 \\
\hline Alto & 10 & 26 \\
\hline
\end{tabular}

\section{Índice de Receptor Soluble de Transferrina}

Todos los hombres presentaron un índice de receptor soluble de transferrina normal, mientras en la población femenina, el 2.2\% (1/46) presentó deficiencia de hierro en estadio I, el $41.3 \%$ (19/46) deficiencia de hierro estadio II y el 8.7\% (4/46) posible deficiencia de hierro sin anemia y un 47.8\% (21/46) fueron normales, Tabla 6.

Tabla 6. Resultados Índice de Receptor Soluble de Transferrina en la muestra estudiada.

\begin{tabular}{|lcc|}
$\begin{array}{l}\text { Îndice de Receptor } \\
\text { Soluble de Transferrina }\end{array}$ & Hombres \% & Mujeres \% \\
\hline Normal & 100 & 47,8 \\
\hline DEF I & & 2,2 \\
\hline DEF II & & 41,3 \\
\hline $\begin{array}{l}\text { Posible deficiencia } \\
\text { de hierro sin anemia }\end{array}$ & 8,7 \\
\hline
\end{tabular}




\section{Fracciones de hemoglobina}

El $83.9 \%(46 / 56)$ de la población presenta hemoglobina A, el 3.6.\% (2/56), aumento en la hemoglobina A2 y hemoglobina $\mathrm{F}$ que se relaciona con un posible rasgo talasémico, el $10.7 \%(6 / 56)$ presentan hemoglobina AS, relacionándolo con rasgo falciforme y el 1.8\% (1/56), presenta hemoglobina $\mathrm{AC}$, que se relaciona con rasgo hemoglobina AC.

Tabla 7. Fracciones de hemoglobina en la muestra estudiada.

\begin{tabular}{|lc|}
\hline \multicolumn{2}{|c|}{ Hemoblobinas } \\
\hline Hb A & Porcentaje \\
\hline Hb A Hb A2 $\uparrow$ HB Fetal $\uparrow$ & 83,9 \\
\hline Hb A Hb S & 3,6 \\
\hline Hb A Hb C & 10,7 \\
\hline
\end{tabular}

\section{Discusión}

Durante el desarrollo del presente proyecto se encontró que en Colombia existen pocos registros sobre la incidencia de anemias debidas a alteración de la molécula de hemoglobina en comunidades afrodescendientes, por ello, este trabajo constituye un apoyo a esta población en la ciudad de Cali, permitiendo la implementación de programas en salud diseñados específicamente para la población afrodescendiente (1-9).

En el trabajo de campo, el grupo de investigación observó que los habitantes del Distrito de Agua Blanca de la ciudad de Cali viven en la periferia de la ciudad, en zonas de invasión, en condiciones de pobreza, estos aspectos conllevan a que su calidad de vida sea precaria y sus condiciones de salubridad sean deficientes (5).

En Colombia existen regiones con alta incidencia de población afrodescendiente, esta investigación presenta datos de cerca de un $20 \%$ de la población de estudio con deficiencia de hierro (10-14) y alteraciones genéticas en la hemoglobina (2). Aunque los resultados demuestran rasgos falciformes, $\mathrm{HbC}$ y posibles talasemias, es importante informar a las entidades de salud regionales con el objetivo de establecer estrategias para la identificación temprana de estas alteraciones más teniendo en cuenta los índices de desplazamientos actuales y los costos que implica el manejo de personas homocigotas.

La población masculina no presentó valores de ferritina bajos, mientras que el 32\% (15/46) mujeres presentaron valores bajos; en cuanto a la correlación con el índice de receptor soluble de transferrina, los hombres fueron normales, mientras que en las mujeres el 2.2\% (1/46) presentaron deficiencia de hierro en estadio I, el 41.3\% (19/46), deficiencia de hierro en estadio II y el $8.7 \%$ (4/46) posible deficiencia de hierro sin anemia, de las dos mujeres que presentaron anemia, en una de ellas no ésta asociado ni a niveles bajos de ferritina ni a alteraciones en la cadena de la hemoglobina, y la otra presentó anemia en estadio II y rasgo falciforme $(9,11,12)$.

El 14.3\% (8/56) fueron positivos para proteína C reactiva, lo que se relaciona con posibles procesos inflamatorios o infecciosos, agudos o crónicos, se sugiere hacer seguimiento para determinar las causas de la elevación de esta proteína de fase aguda.

$\mathrm{Al}$ realizar el estudio por electroforesis de hemoglobina, el $83.9 \%(46 / 56)$ de la población presenta hemoglobina A, el $3.6 \%(2 / 56)$ aumento de la hemoglobina A2 y hemoglobina fetal que se relaciona con un posible rasgo talasémico, el $10.7 \%$ (6/56), presentaron hemoglobina AS relacionado con rasgo falciforme, y el $1.8 \%(1 / 56)$, presentó hemoglobina $\mathrm{AC}$, que se relaciona con rasgo hemoglobina AC (15).

Los resultados del estudio fueron enviados a los representantes de la comunidad en estudio, quienes se comprometieron a presentarlos a los entes respectivos de salud para hacer la intervención necesaria de acuerdo a lo reportado. 


\section{Referencias}

1. De Maeyer E, Adiels-Tegman M. The prevalence of anaemia in the world. World Health Stat Q. 1985; 38:302-16.

2. Durán $\mathrm{Cl}$, Morales Ol, Echeverri Sj, Isaza M. Haplotipos Del Gen De La Globina Beta En Portadores De Hemoglobina S En Colombia. Laboratorio Clínico, Clínica Colsanitas. Biomédica. 2012;32:103-11.

3. María C. Mondragón A. Capítulo 11 Anemia Falciforme Y Talasemia. En: Francisco Cuellar A, Francisco Falabella F. Fundamentos De Medicina. Hematología. Volumen 6. Colombia. Corporación Para Investigaciones Biológicas. 2004. P. 91

4. Punnonen K, Irjala K, Rajamäki A. Serum Transferrin Receptor and Its Ratio to Serum Ferritin in the Diagnosis of Iron Deficiency. Blood. 1997; 89(3): 1052-7.

5. Castillo M, Mora A, Donato K, Pérez F, Tapiero M. Identification of iron deficiency risk by index soluble transferrin receptor-log ferritin in African descent men living at San Basilio de Palenque, Cartagena de Indias, T. and C. D, Bolivar, Colombia. NOVA 2010; 54-62.

6. Alvear Ciro Cesar, Barboza Miriam, Viola Maricela, Moneriz Carlos, Araque Luz Marina. Pilot Study Of Hemoglobinopathies In Newborns Of The Rafael Calvo Maternity Clinic Of Cartagena, Colombia. Colomb. Med. [Serial On The Internet]. 2012 July; 43(3): 196-199. Available From: Http://Www.Scielo.Org.Co/Scielo.Php?Script=Sci_ Arttext\&Pid=S1657-95342012000300004\&Lng=En.

7. Restrepo F, Natalia Loaiza, Marcela Arrubla, Sandra Patricia Cossio, Jaime Ordoñez Tamización Neonatal Por Cromatografía De Alta Resolución Para El Diagnóstico De Anemia De Células Falciformes Y Otras Hemoglobinopatías En Medellín, Colombia ISSN 0120-4912. 2011; 43(4).
8. Gibbons A (2007). "American Association of Physical Anthropologists meeting. European skin turned pale only recently, gene suggests". Science,316 (5823): 364. DOI:10.1126/ science.316.5823.364a

9. Suominen P, Punnonoen K, Rajamäki A. Serum transferrin receptor and transferrin receptor-ferritin. Index Identify Healthy Subjects with subclinical iron deficits. Blood. 1998; 92 (8): 2934-39.

10. Cook JD, Lipschitz DA, Miles LE, Finch CA. Serum ferritin as measure of iron stores in normal subjets. Am J Clin Nutr. 1974; 27:681-7.

11. Ahluwalia N, Lönnerdal B, Lorenz S, Allen L. Spot ferritin assay for serum simples dried on filter paper. Am J Clin Nutr. 1998; 67:88-92.

12. Kato J, Kobune M, Kohgo Y, Fujikawa R, Takimoto R, Torimoto Y. Ratio of Transferrin (Tf) to Tf-Receptor Complex in Circulation Differs Depending on Tf Iron Saturation. Clin Chem. 2002; 48:181-3.

13. Rimon E, Levy S, Sapir A, Gelzer G, Peled R, Ergas D, et al. Diagnosis of iron deficiency anemia in the elderly by transferrin receptor - ferritin index. Arch Intern Med 2002; 162: 445-9.

14. Choi J. Sensitivity, Specificity, and Predictive Value of Serum Soluble Transferrin Receptor at Different Stages of Iron Deficiency. Ann Clin \& Lab Sci. 2005; 35: 435-39.

15. Svarch E. Fisiopatología De La Drepanocitosis. Revista Cubana De Hematología, Inmunología Y Medicina Transfusional. 2009; 25(1):1-15. 